\title{
Circadian rhythms and metabolic disorders: an epigenetic approach
}

\section{Dragos I Mosneagu, Hashmeena Sanghani, Sridhar Vasudevan}

Department of Pharmacology, University of Oxford, UK

Circadian rhythms are an organism's biological way of anticipating pivotal changes in bodily functions by using the timeof-the-day as their main input. Genetics, as well as environmental factors are key elements responsible for the modulation of circadian rhythms. Any disruption to either of these factors can lead to sleep and circadian rhythm disorders (SCRD) as well as associated pathologies. Although intense efforts have been directed towards understanding the genetic bases of the circadian rhythm physiology and pathology, little is known about the pivotal role played by environmental factors. Food intake, activity and age, although known to entrain the circadian genes' expression, are elusive factors with regards to their mechanism of action (MOA). Epigenetics is thought to be fundamental in the biology of circadian rhythms and it is hypothesised to be one of the key mediators linking circadian rhythms to external factors, such as light, temperature, stress, drugs, hormones, food, and age. Moreover, the interaction between circadian rhythms and metabolic physiology has shown to display an intricate epigenetic MOA, all mechanisms worth shedding light upon.

We have pinpointed a novel class of epigenetic modulators that showed to have a phenotypic circadian effect. By increasing amplitude of circadian gene expression, we hypothesise this class of molecules will help better delineate the time-dependent clockwork of bodily functions. More specifically, my focus is to decipher the intricate mechanism that regulates circadian rhythms and metabolism.

It is well established that $\mathrm{NAD}+$ decrements occur in various tissues during ageing and that pharmacological interventions that increase cellular NAD+ levels might retard ageing aspects. However, little is known about the effects of NAD+ in circadian rhythms. We showed our novel epigenetic modulators to rely on Sirt1, a histone deacetylase, and other enzymes important to the biosynthesis of NAD+, a vital cofactor that can rewire metabolism, activate Sirtuins and potentially modulate ageing.

By further studying this class of molecules, we address the shortfall of understanding that connects circadian rhythm disruptions with metabolic disorders and may provide unique and novel insights into the development of new therapeutic strategies to control or combat metabolic disorders such as T2DM and clinical obesity. 